\title{
Open-Source Visualization of 3D Data: From Tomography to Spectroscopy
}

\author{
Robert Hovden ${ }^{1}$, Paul Cueva ${ }^{1}$, David A. Muller ${ }^{1,2}$
}

1. School of Applied and Engineering Physics, Cornell University, Ithaca, NY

2. Kavli Institute at Cornell for Nanoscale Science, Ithaca, New York

The deluge of digital data acquired by the modern electron microscopist comes with added challenges in data visualization. Data is no longer restricted to a single two-dimensional (2D) image. Even at the atomic-scale, digital images are now collected as through-focal stacks, time series, spectroscopic maps, and tomographic reconstructions. These three-dimensional (3D) datasets require extensive software tools for analysis and visualization. This is accomplished either through self-written code, commercial software, or open-source tools. However, commercial software can be mysterious to its inner workings and prohibitively expensive-especially for user-facilities where users wish to process data off-site. Free open-source software can provide accessible, transparent solutions for analysis and visualization of 3D electron microscopy data. Here we demonstrate the use and extendibility of open-source tools for the visualization of 3D datasets, with focus on spectroscopic mapping and tomographic reconstructions.

Visualizing a 3D tomogram requires software that can utilize large quantities of memory and processing resources to render and manipulate 3D objects. Fortunately, free open-source packages are available [1-3]. Perhaps the most robust open-source 3D visualization tool is ParaView, with a GUI workflow built on the visualization toolkit (VTK) framework (Fig. 1) [1]. Objects can be rendered as shaded iso-surfaces (Fig a,b,d) or volumetric projections. Multiple datasets, colormaps, and other visualization settings can be used in combination and these objects can be rotated, sliced, and animated into video files. Figure 1 shows Paraview visualization of a hyperbranched $\mathrm{Co}_{2} \mathrm{P}$ nanocrystal reconstructed from scanning transmission electron microscope data [4]. Data collected can be further analyzed through histograms, multicorrelative statistics, and filters - to name a few. And because it is open source, new algorithms can be developed into Paraview or more readily implemented through its Python scripting interface.

Spectroscopic mapping has become a common 3D dataset that accompanied the high-beam currents of aberration-corrected microscopes. This includes electron energy loss spectroscopy, x-ray emmission, and cathodoluminescence. Like tomography, these datasets can be quite large ( $>1 \mathrm{~GB}$ ). Few open-source tools exists for processing an electron microscopist's spectroscopic datacubes $[5,6]$. More recently, the 64-bit Cornell Spectrum Imager (CSI) (Fig. 2) developed as a universal spectral analysis tool to run on all computers, minimize training, and provide transparency to its algorithms and approximations. In CSI, spectroscopic datasets become easily browseable in the spacial and spectroscopic dimensions (Fig 2b,c). Spectrum backgrounds can be modeled and subtracted, peaks can be extracted by integration, and the most influential components can be mapped with principle component analysis [5]. Built as a plugin for the open-source ImageJ platform, it comes with a multitude of image analysis functionality [7]. The code can be modified, extended, and combined with existing ImageJ libraries.

Micrographs are no longer 2D images developed onto film - the skill and time once spent on darkroom techniques has translated into digital processing. With it, the reliance on software has increased. Software highlighted here mentions a few well-developed tools, but only represents a fraction of all open-source solutions for the electron microscopist.

[1] A. Henderson, ParaView Guide, A Parallel Visualization Application. Kitware Inc., 2007

[2] "VisIt Visualization Environment." Lawrence Livermore National Lab, www.llnl.gov/visit/ 
[3] J Schindelin, et al. Nature Methods 9, 676-682 (2012)

[4] H. Zhang, et al Nano Lett. 11 (2011) 188-197

[5] P Cueva, R Hovden, JA Mundy, HL Xin, and DA Muller, Microsc Microanal 18 (2012) 667-75.

[6] J Verbeeck and S Van Aert, Model Ultramicroscopy 101 (2004) 207-24 / www.eelsmodel.ua.ac.be

[7] Schneider, C.A., Rasband, W.S., Eliceiri, K.W. Nature Methods 9, 671-675, 2012
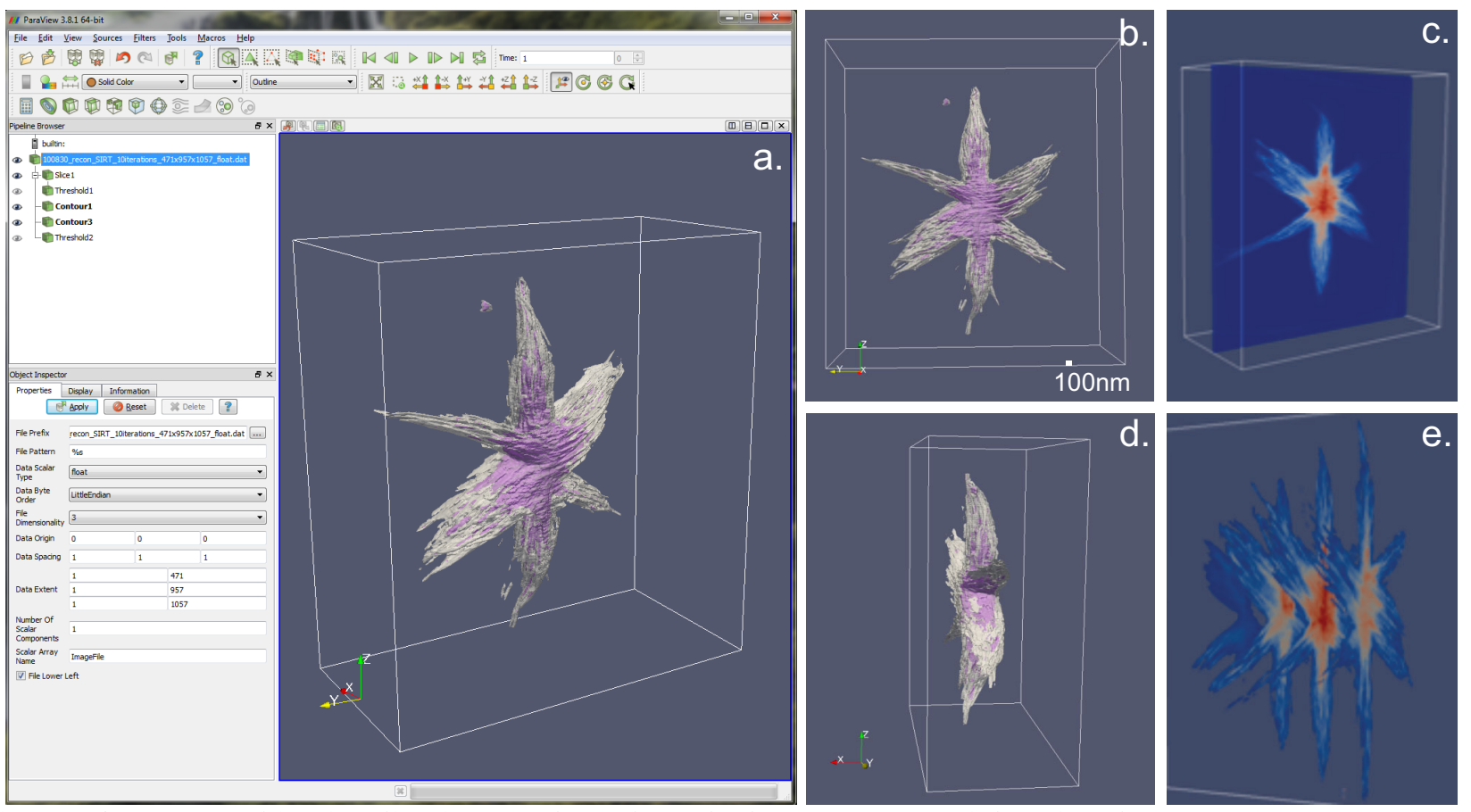

Figure 1 | Tomographic visualization of a hyperbranched Co2P nanoparticle using the open-source Paraview software tools (v. 3.8.1). Isosurface renderings (a,b,d) show star shaped structure (white) and its core region (purple). Slices taken through the object illustrate the internal structure of the particle (c,e).

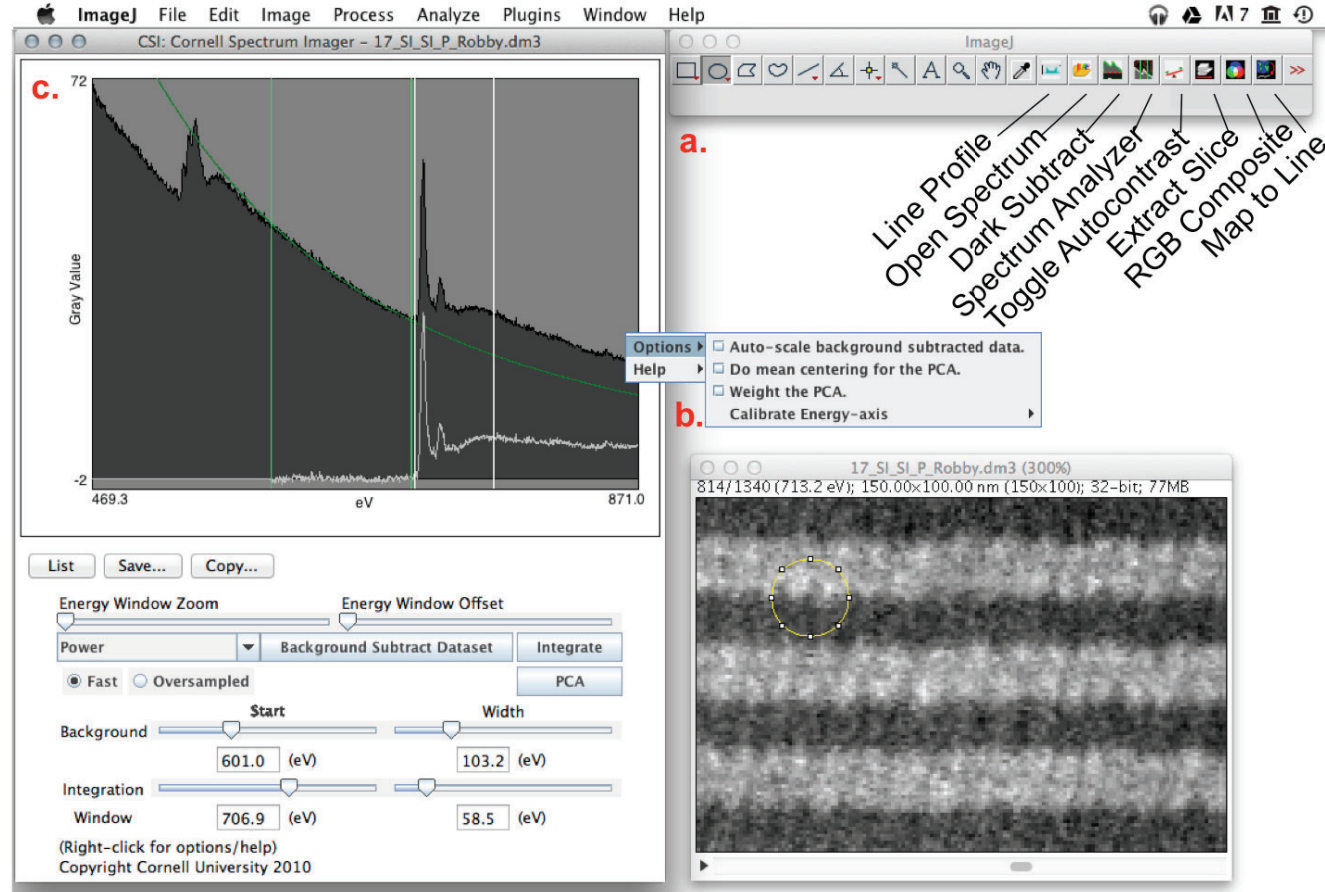

Figure 2 | Screenshot of CSI software (v1.4) being used to analyze EELS spectral map data. c) Spectrum analyzer showing background subtraction, and (d) the spectral map data displayed at a particular energy loss. 